\title{
Scattering Analysis by the Multiresolution Time-Domain Method Using Compactly Supported Wavelet Systems
}

\author{
Traian Dogaru and Lawrence Carin \\ Department of Electrical and Computer Engineering \\ Duke University \\ Box 90291 \\ Durham, NC 27708-0291
}

\begin{abstract}
We present a formulation of the multiresolution time-domain (MRTD) algorithm using scaling and one-level wavelet basis functions, for orthonormal Daubechies and biorthogonal Cohen-Daubechies-Feauveau (CDF) wavelet families. We address the issue of the analytic calculation of the MRTD coefficients. This allows us to point out the similarities and the differences between the MRTD schemes based on the aforementioned wavelet systems and to compare their performances in terms of dispersion error and computational efficiency. The remainder of the paper is dedicated to implementation of the CDF-MRTD method for scattering problems. We discuss the approximations made in implementing material inhomogeneities and validate the method by numerical examples.
\end{abstract}

\section{Introduction}

The multiresolution time-domain (MRTD) method [1-5] has recently emerged as an efficient tool for time-domain electromagnetic field analysis, with applications including microwave cavities and circuits $[1,3,4]$ as well as scattering by general targets [2]. In many cases, MRTD can save important computational resources, as compared to the traditional FDTD method [6], without sacrificing solution accuracy. The main mechanisms by which MRTD achieves computational efficiency are the higher-order accuracy in the spatial finite-difference approximations and the multi-resolution partitioning of the computational domain. With regard to the latter, denser resolution is employed in zones with relatively fast spatial field variation, 
while applying a lower-resolution representation in slowly varying regions.

In the existing literature on MRTD, the basis functions of choice have been the BattleLemarie wavelet family [1], the Haar wavelet family [2], the Daubechies scaling functions [3,4] and the biorthogonal Cohen-Daubechies-Feauveau (CDF) wavelet systems [5]. In a previous paper [5], we demonstrated the advantages of the CDF wavelet systems vis-à-vis the BattleLemarie or Haar wavelet families. In general, the choice of basis functions with minimal support leads to a small stencil size and a large Courant stability limit [5], which in turn yield a computationally efficient scheme. Another advantage of the reduced support of the basis functions becomes apparent as we discuss the implementation of material boundaries.

In the current paper, we focus on the compactly supported wavelets from the orthonormal Daubechies and biorthogonal CDF families. First, we extend the Daubechies MRTD scheme to include first-level wavelet functions (Sec. II). After reviewing the CDF-MRTD formulation (Sec. III), we present an algorithm for the analytic computation of the MRTD coefficients, which is faster, more accurate and more general than the numerical integration utilized in [1-5]. We compare the MRTD schemes based on low-order orthonormal Daubechies and biorthogonal CDF wavelet systems in terms of numerical performance in Sec. IV. In order to implement the CDF-MRTD scheme for scattering problem, we discuss modeling the material inhomogeneities and the plane-wave incident field (Sec. V). In Sec. VI we present numerical results on two-dimensional scattering configurations, emphasizing the efficiency of the method. We draw conclusions in Sec. VII.

\section{MRTD Based on Orthonomal Daubechies Wavelets}

\section{A. Formulation}

The MRTD algorithm based on compactly supported orthonormal Daubechies scaling functions was presented in $[3,4]$. Here, we extend the algorithm to include one-level wavelet 
functions, from which we obtain a multi-resolution algorithm. Restricting the presentation to one dimension (for simplicity), the field expansion can be written as:

$$
\begin{gathered}
E_{z}(x, t)=\sum_{k, m=-\infty}^{\infty}\left[E_{k, m}^{\Phi} \Phi_{m}(x)+E_{k, m}^{\Psi} \Psi_{m}(x)\right]_{k}(t) \\
H_{y}(x, t)=\sum_{k, m=-\infty}^{\infty}\left[H_{k, m}^{\Phi} \Phi_{m+1 / 2}(x)+H_{k, m}^{\Psi} \Psi_{m+1 / 2}(x)\right] h_{k+1 / 2}(t)
\end{gathered}
$$

Here, we denote by $\Phi_{m}$ and $\Psi_{m}$ the scaling and the wavelet function, respectively, displaced by $m$ units. For time discretization we use rectangular pulses $h_{k}(t)$, where $k$ represents the shift in time units. The MRTD magnetic-field update equations are:

$$
\begin{gathered}
H_{k, m}^{\Phi}=H_{k-1, m}^{\Phi}+\frac{\Delta t}{\mu \Delta x}\left(\sum_{i=-n_{a}+1}^{n_{a}} a(i) E_{k, m+i}^{\Phi}+\sum_{i=-n_{c}+1}^{n_{c}} c(i) E_{k, m+i}^{\Psi}\right) \\
H_{k, m}^{\Psi}=H_{k-1, m}^{\Psi}+\frac{\Delta t}{\mu \Delta x}\left(\sum_{i=-n_{d}+1}^{n_{d}} d(i) E_{k, m+i}^{\Phi}+\sum_{i=-n_{b}+1}^{n_{b}} b(i) E_{k, m+i}^{\Psi}\right) \\
E_{k+1, m}^{\Phi}=E_{k, m}^{\Phi}+\frac{\Delta t}{\varepsilon \Delta x}\left(\sum_{i=-n_{a}+1}^{n_{a}} a(i) H_{k, m+i-1}^{\Phi}+\sum_{i=-n_{c}+1}^{n_{c}} c(i) H_{k, m+i-1}^{\Psi}\right) \\
E_{k+1, m}^{\Psi}=E_{k, m}^{\Psi}+\frac{\Delta t}{\varepsilon \Delta x}\left(\sum_{i=-n_{d}+1}^{n_{d}} d(i) H_{k, m+i-1}^{\Phi}+\sum_{i=-n_{b}+1}^{n_{b}} b(i) H_{k, m+i-1}^{\Psi}\right)
\end{gathered}
$$

where $\quad a(i)=\int \frac{\partial \Phi(x)}{\partial x} \Phi(x+i-1 / 2) d x \quad, \quad b(i)=\int \frac{\partial \Psi(x)}{\partial x} \Psi(x+i-1 / 2) d x$ $c(i)=\int \frac{\partial \Psi(x)}{\partial x} \Phi(x+i-1 / 2) d x, d(i)=\int \frac{\partial \Phi(x)}{\partial x} \Psi(x+i-1 / 2) d x$ are the MRTD coefficients.

\section{B. Calculation of the MRTD coefficients}

In previous MRTD papers based on Daubechies scaling functions (as well as other 
MRTD schemes), the MRTD coefficients $a, b, c$ and $d$ were computed by numeric integration (usually in the Fourier domain). However, based on the algorithm presented in [7], these coefficients can be derived analytically in the case of compactly supported wavelet systems, starting directly from the scaling/wavelet filter coefficients [8], without the need to explicitly compute the scaling/wavelet functions (or their Fourier counterparts). Using the definition of the scaling and wavelet filter coefficients:

$$
\begin{aligned}
& \Phi(x)=\sqrt{2} \sum_{k=0}^{L-1} h_{k} \Phi(2 x-k) \\
& \Psi(x)=\sqrt{2} \sum_{k=0}^{L-1} g_{k} \Phi(2 x-k)
\end{aligned}
$$

we introduce the following correlation coefficients: $\alpha_{n}=2 \sum_{k} h_{k} h_{k+n}, \quad \beta_{n}=2 \sum_{k} g_{k} g_{k+n}$, $\gamma_{n}=2 \sum_{k} h_{k} g_{k+n}, \delta_{n}=2 \sum_{k} g_{k} h_{k+n}$, with $n=-L+2, \ldots L-2$, with the sums over $k$ running from $\max (0,-n)$ and $\min (L-1, L-1-n)$. In the above, $L$ is the length of the non-zero scaling filter coefficient sequence.

We start with the calculation of the coefficients $r(i)=\int \frac{\partial \Phi(x)}{\partial x} \Phi(x-i) d x$, as described in [7]. Specifically, these coefficients must satisfy the following linear system:

$$
\begin{gathered}
r(i)=\sum_{n=-L+2}^{L-2} \alpha_{n} r(2 i+n) \\
\sum_{i=-L+2}^{L-2} i r(i)=-1
\end{gathered}
$$

Notice that one of the equations in (4.a) is redundant and that only a finite number of coefficients $r(i)$ are non-zero, with indices running from $-L+2$ to $L-2$. Once these coefficients were found, the MRTD coefficients are given by the following expressions: 


$$
\begin{aligned}
& a(i)=-\sum_{n=-L+1}^{L-1} \alpha_{n} r(2 i+n-1) \\
& b(i)=-\sum_{n=-L+1}^{L-1} \beta_{n} r(2 i+n-1) \\
& c(i)=-\sum_{n=-L+1}^{L-1} \gamma_{n} r(2 i+n-1) \\
& d(i)=-\sum_{n=-L+1}^{L-1} \delta_{n} r(2 i+n-1)
\end{aligned}
$$

Again, the number of non-zero MRTD coefficients is finite and we can establish the following symmetry relationships: $r(-i)=r(i), a(-i)=-a(i+1), b(-i)=-b(i+1), c(-i)=-d(i+1)$.

\section{MRTD Based on Biorthogonal CDF Wavelets}

\section{A. Formulation}

The biorthogonal CDF-MRTD scheme was introduced and analyzed in detail in [5]. In this paper, we consider only the symmetric CDF $(2, M)$ families of wavelets (where $M$ is half the support length of the scaling function), for the purpose of comparison with the orthonormal Daubechies wavelet families. The field expansions in one dimension can be written as:

$$
\begin{gathered}
E_{z}(x, t)=\sum_{k, m=-\infty}^{\infty}\left[E_{k, m}^{\Phi} \tilde{\Phi}_{m}(x)+E_{k, m}^{\Psi} \tilde{\Psi}_{m}(x)\right]_{k}(t) \\
\left.H_{y}(x, t)=\sum_{k, m=-\infty}^{\infty}\left[H_{k, m}^{\Phi} \tilde{\Phi}_{m+1 / 2}(x)+H_{k, m}^{\Psi} \tilde{\Psi}_{m-1 / 2}(x)\right]\right]_{k+1 / 2}(t)
\end{gathered}
$$

The above notation assumes that both $\Psi(x)$ and $\tilde{\Psi}(x)$ peak at $x=1 / 2$. We obtain the following magnetic field update equations: 


$$
\begin{gathered}
H_{k, m}^{\Phi}=H_{k-1, m}^{\Phi}+\frac{\Delta t}{\mu \Delta x}\left(\sum_{i=-n_{a}+1}^{n_{a}} a(i) E_{k, m+i}^{\Phi}+\sum_{i=-n_{c}}^{n_{c}} c(i) E_{k, m+i}^{\Psi}\right) \\
H_{k, m}^{\Psi}=H_{k-1, m}^{\Psi}+\frac{\Delta t}{\mu \Delta x}\left(\sum_{i=-n_{d}}^{n_{d}} d(i) E_{k, m+i}^{\Phi}+\sum_{i=-n_{b}+1}^{n_{b}} b(i) E_{k, m+i-1}^{\Psi}\right) \\
E_{k+1, m}^{\Phi}=E_{k, m}^{\Phi}+\frac{\Delta t}{\varepsilon \Delta x}\left(\sum_{i=-n_{a}+1}^{n_{a}} a(i) H_{k, m+i-1}^{\Phi}+\sum_{i=-n_{c}}^{n_{c}} c(i) H_{k, m+i}^{\Psi}\right) \\
E_{k+1, m}^{\Psi}=E_{k, m}^{\Psi}+\frac{\Delta t}{\varepsilon \Delta x}\left(\sum_{i=-n_{d}}^{n_{d}} d(i) H_{k, m+i}^{\Phi}+\sum_{i=-n_{b}+1}^{n_{b}} b(i) H_{k, m+i}^{\Psi}\right)
\end{gathered}
$$

where $\quad a(i)=\int \frac{\partial \tilde{\Phi}(x)}{\partial x} \Phi(x+i-1 / 2) d x \quad, \quad b(i)=\int \frac{\partial \tilde{\Psi}(x)}{\partial x} \Psi(x+i-1 / 2) d x \quad$ $c(i)=\int \frac{\partial \tilde{\Psi}(x)}{\partial x} \Phi(x+i-1 / 2) d x, d(i)=\int \frac{\partial \tilde{\Phi}(x)}{\partial x} \Psi(x+i+1 / 2) d x$.

\section{B. Calculation of the MRTD coefficients}

The calculation of the MRTD coefficients in the case of the CDF $(2, M)$ biorthogonal wavelet expansion is similar to that described for the Daubechies wavelet-based MRTD scheme. In this case, we define:

$$
\begin{gathered}
\Phi(x)=\sqrt{2} \sum_{k=-L / 2}^{L / 2} h_{k} \Phi(2 x-k) \\
\Psi(x+1 / 2)=\sqrt{2} \sum_{k=-L / 2}^{L / 2} g_{k} \Phi(2 x-k) \\
\tilde{\Phi}(x)=\sqrt{2} \sum_{k=-L / 2}^{L / 2} \tilde{h}_{k} \tilde{\Phi}(2 x-k) \\
\tilde{\Psi}(x+1 / 2)=\sqrt{2} \sum_{k=-L / 2}^{L / 2} \tilde{g}_{k} \tilde{\Phi}(2 x-k)
\end{gathered}
$$


In the above, $L+1=2 M+1$ indicates the length of the $h_{k}$ filter-coefficient sequence (notice that we use a symmetric form of this sequence, with indices running from $-L / 2$ to $L / 2$ ). We also bring the other filter coefficient sequences $g_{k}, \tilde{h}_{k}$ and $\tilde{g}_{k}$ to the same length, by zero-padding where needed. The correlation coefficients are defined as: $\alpha_{n}=2 \sum_{k} h_{k} \tilde{h}_{k+n}, \quad \beta_{n}=2 \sum_{k} g_{k} \widetilde{g}_{k+n}$, $\gamma_{n}=2 \sum_{k} h_{k} \tilde{g}_{k+n}, \delta_{n}=2 \sum_{k} g_{k} \tilde{h}_{k+n}$, with $n=-L, \ldots L$, and the sums over $k$ running from max($L / 2,-L / 2+n)$ and $\min (L / 2-n, L / 2)$. One can show that the coefficients $r(i)=\int \frac{\partial \tilde{\Phi}(x)}{\partial x} \Phi(x-i) d x$ formally satisfy the same set of equations as indicated in (4.a) and (4.b). From here, the MRTD coefficients are computed as following:

$$
\begin{array}{r}
a(i)=-\sum_{n=-L}^{L} \alpha_{n} r(2 i+n-1) \\
b(i)=-\sum_{n=-L}^{L} \beta_{n} r(2 i+n-1) \\
c(i)=-\sum_{n=-L}^{L} \gamma_{n} r(2 i+n) \\
d(i)=-\sum_{n=-L}^{L} \delta_{n} r(2 i+n)
\end{array}
$$

The following symmetry relationships can be established: $r(-i)=r(i), a(-i)=-a(i+1)$, $b(-i)=-b(i+1), c(-i)=-c(i), d(-i)=-d(i)$.

\section{Analysis of Daubechies-4 and CDF (2,2) MRTD Schemes}

In this section we explicitly compute the MRTD coefficients for the Daubechies-4 wavelet family (i.e., $L=4)$ and for the $\operatorname{CDF}(2,2)$ wavelet family. The values of the non-zero correlation coefficients $\alpha, \beta, \gamma, \delta$, as well as the MRTD coefficients $a, b, c, d$ are given in Table I. As expected, the $a$ coefficients are the same for the two wavelet families, which means that the 
MRTD schemes based only on scaling functions from these families are completely equivalent. However, the $c$ and $d$ coefficients are different for the two wavelet families, therefore the two MRTD schemes are not identical at the wavelet-expansion level. Similar conclusions can be drawn in general when one compares the MRTD schemes resulting from expansions in terms of Daubechies- $(M+2)$ and $\operatorname{CDF}(2, M)$ basis functions.

We can also compare other aspects related to the computational efficiency and performance of the MRTD schemes based on Daubechies- $(M+2)$ or CDF $(2, M)$ wavelet families. The stability analysis [5] shows that the Courant numbers at the stability limit are not identical, but very close for the two schemes. In particular, for Daubechies-4, this limit is 0.6040859 , whereas for the $\operatorname{CDF}(2,2)$ it is 0.6045534 (both scaling and one level wavelet functions are taken in the expansion). Also, the dispersion curves [5] are very similar, although not identical. In Fig. 1 we plot the phase error (in degrees per wavelength) measured as $360\left(\frac{\lambda_{\text {continuous }}}{\lambda_{\text {discrete }}}-1\right)$ (the expression involves the theoretical and numerical wavelengths), as a function of the number of sampling points per wavelength, in one-dimensional propagation. Three pairs of schemes were compared (Daubechies-4 with CDF (2,2), Daubechies-6 with CDF $(2,4)$ and Daubechies-8 with CDF $(2,6)$ ), while the Courant number was taken $95 \%$ of the stability limit for each scheme. We notice again the similar performance of Daubechies- $(M+2)$ and $\operatorname{CDF}(2, M)$ schemes (the curves are not identical, but they cannot be distinguished as plotted in Fig. 1).

Finally, the stencil size of the numerical scheme (given by $n_{a}, n_{b}, n_{c}, n_{d}$ in equations (2) or (7)) determines the number of floating point operations executed at each time step. A low stencil size is desirable in order to reduce the CPU time required by the algorithm. The values of $n_{a}, n_{b}$, $n_{c}$ and $n_{d}$ are given in Table II for the MRTD schemes based on the same three pairs of expansions. Once again, we notice very similar characteristics between the Daubechies- $(M+2)$ and the $\operatorname{CDF}(2, M)$ schemes. 


\section{MRTD Implementation of Scattering Problems}

\section{A. Plane-wave incident field implementation}

Modeling of electromagnetic scattering problems with distant sources involves the implementation of the incident field as a plane wave. For the MRTD algorithm, the incident field can be implemented in a manner similar to as in the traditional FDTD scheme, that is, splitting the computational domain into two regions: one of total fields and the other of scattered fields only, separated by a connecting surface [6]. The incident field is introduced as a 'boundary condition' at this surface, in order to enforce the continuity of the tangential field components. This idea was used in [2], where the MRTD method based on the Haar wavelet system was utilized in modeling scattering problems. The extension to other wavelet bases is straightforward. If the update equations involve more than one field scaling/wavelet coefficient on each side of the current point (which now is placed next to the connecting surface), all the field coefficients placed on the other side of the connecting surface must be adjusted by the appropriate incident field scaling/wavelet coefficients, such that we preserve the consistency of the equations.

\section{B. Modeling material inhomogeneities}

The treatment of inhomogeneous media configurations in the context of MRTD poses significant problems, because the material properties, as functions of space, introduce coupling between adjacent basis functions. In [1], the inhomogeneities are treated rigorously, using a matrix formulation. The authors of [3] and [4] make use of the shifted interpolation property of the Daubechies scaling functions in order to simplify the equations. However, their formulation is valid only as an approximation. In this section, we present the exact formulation of the CDFMRTD equations at a dielectric interface and discuss the approximations that can be made in order to simplify the formulation. 
Let us assume a one-dimensional expansion in terms of scaling functions from the CDF $(2,2)$ wavelet family. In Fig. 2 , the interface is placed at the coordinate $n \Delta x$, and the permittivities on the two sides are $\varepsilon_{1}$ and $\varepsilon_{2}$, respectively. The scaling and dual scaling basis functions which get coupled through $\varepsilon(x)$ are schematically drawn in the same figure. The cross-terms between MRTD equations appear when the integrals

$$
\varepsilon_{i, j}^{\Phi \Phi}=\int \varepsilon(x) \Phi_{n+i}(x) \tilde{\Phi}_{n+j}(x) d x
$$

are non-zero. In the case of $\operatorname{CDF}(2,2)$ scaling functions, this occurs when $j=0, i=-1,0,1$ (see Fig. 2). Therefore, discretization of the equation $\varepsilon \frac{\partial E_{z}}{\partial t}=\frac{\partial H_{y}}{\partial x}$ about the point of coordinate $n \Delta x$ leads to a set of three coupled MRTD equations:

$$
\begin{array}{rr}
\varepsilon_{-1,-1}^{\Phi \Phi}\left(E_{k+1, n-1}^{\Phi}-E_{k, n-1}^{\Phi}\right)+\varepsilon_{-1,0}^{\Phi \Phi}\left(E_{k+1, n}^{\Phi}-E_{k, n}^{\Phi}\right) & =\frac{\Delta t}{\Delta x}\left(\frac{\partial H_{y}}{\partial x}\right)_{k, n-1}^{\Phi} \\
\varepsilon_{0,0}^{\Phi \Phi}\left(E_{k+1, n}^{\Phi}-E_{k, n}^{\Phi}\right) & =\frac{\Delta t}{\Delta x}\left(\frac{\partial H_{y}}{\partial x}\right)_{k, n}^{\Phi} \\
\varepsilon_{1,0}^{\Phi \Phi}\left(E_{k+1, n}^{\Phi}-E_{k, n}^{\Phi}\right)+\varepsilon_{1,1}^{\Phi \Phi}\left(E_{k+1, n+1}^{\Phi}-E_{k, n+1}^{\Phi}\right) & =\frac{\Delta t}{\Delta x}\left(\frac{\partial H_{y}}{\partial x}\right)_{k, n+1}^{\Phi}
\end{array}
$$

We have used simplified notation for the curl terms on the right hand side, meaning the scalinglevel discretized version of the spatial derivative of the magnetic field component, at the current point (e.g., $n$ ) and the current time step $k$. With the notation $[\varepsilon]=\left[\begin{array}{ccc}\varepsilon_{-1,-1}^{\Phi \Phi} & \varepsilon_{-1,-0}^{\Phi \Phi} & 0 \\ 0 & \varepsilon_{0,0}^{\Phi \Phi} & 0 \\ 0 & \varepsilon_{1,0}^{\Phi \Phi} & \varepsilon_{1,1}^{\Phi \Phi}\end{array}\right]$, we obtain the following matrix equation: 


$$
\left[\begin{array}{c}
E_{k+1, n-1}^{\Phi} \\
E_{k+1, n}^{\Phi} \\
E_{k+1, n+1}^{\Phi}
\end{array}\right]=\left[\begin{array}{c}
E_{k, n-1}^{\Phi} \\
E_{k, n}^{\Phi} \\
E_{k, n+1}^{\Phi}
\end{array}\right]+\frac{\Delta t}{\Delta x}[\varepsilon]^{-1}\left[\begin{array}{c}
\left(\partial H_{y} / \partial x\right)_{k, n-1}^{\Phi} \\
\left(\partial H_{y} / \partial x\right)_{k, n}^{\Phi} \\
\left(\partial H_{y} / \partial x\right)_{k, n+1}^{\Phi}
\end{array}\right]
$$

Notice that $\varepsilon_{-1,-1}^{\Phi \Phi}=\varepsilon_{1}, \quad \varepsilon_{1,1}^{\Phi \Phi}=\varepsilon_{2}$ and $\varepsilon_{0,0}^{\Phi \Phi}=\frac{\varepsilon_{1}+\varepsilon_{2}}{2}$. A similar treatment is obtained by considering lossy medium, with a frequency independent conductivity (see [2]). The formulation can be generalized to any basis of symmetric scaling functions (including higher-order CDF or Battle-Lemarie wavelet families). If the scaling function $\Phi$ has a support of length $2 M$ and the dual scaling function $\tilde{\Phi}$ has a support of length $2 \tilde{M}$, then the $\varepsilon$-matrix has dimensions $(2 P+1) \times(2 P+1)$, where $P=M+\tilde{M}-2$, and a band-diagonal structure, with $P+1$ non-zero diagonals.

In the case where the interface does not coincide with a grid line, as in Fig. 3 (where the boundary falls between the coordinates $n \Delta x$ and $(n+1) \Delta x)$, the formulation is similar, only this time we have four coupled equations (for $\operatorname{CDF}(2,2)$ expansion), involving the field expansion coefficients at points $n-1, n, n+1$ and $n+2$. The $\varepsilon$-matrix has the following structure:

$$
[\varepsilon]=\left[\begin{array}{cccc}
\varepsilon_{-1,0}^{\Phi \Phi} & \varepsilon_{-1,0}^{\Phi \Phi} & \varepsilon_{-1,1}^{\Phi \Phi} & 0 \\
0 & \varepsilon_{0,0}^{\Phi \Phi} & \varepsilon_{0,1}^{\Phi \Phi} & 0 \\
0 & \varepsilon_{1,0}^{\Phi \Phi} & \varepsilon_{1,1}^{\Phi \Phi} & 0 \\
0 & \varepsilon_{2,0}^{\Phi \Phi} & \varepsilon_{2,1}^{\Phi \Phi} & \varepsilon_{2,2}^{\Phi \Phi}
\end{array}\right]
$$

If wavelet functions are considered in the expansion, the situation complicates even further, because of the coupling that appears between the scaling and wavelet functions. Without going into details, we indicate the structure of the $\varepsilon$-matrix in the case of the $\operatorname{CDF}(2,2)$ expansion involving scaling and one-level wavelet functions: 


$$
[\varepsilon]=\left[\begin{array}{ccccccc}
\times & \times & 0 & 0 & \times & \times & 0 \\
0 & \times & 0 & 0 & \times & \times & 0 \\
0 & \times & \times & 0 & \times & \times & 0 \\
0 & \times & 0 & \times & \times & \times & 0 \\
0 & \times & 0 & 0 & \times & \times & 0 \\
0 & \times & 0 & 0 & \times & \times & 0 \\
0 & \times & 0 & 0 & \times & \times & \times
\end{array}\right]
$$

where the $\times$ 's symbolize non-zero entries.

The presence of the matrix equations, although limited to regions close to the interface, introduces extra calculations that reduce the computational efficiency of the algorithm. In theory, one can compute and invert the $\mathcal{\varepsilon}$-matrices for a given media configuration at the beginning of the program, and then add the extra terms to the appropriate equations at each time step (notice that the inverse $\varepsilon$-matrices have identical structures with the original $\varepsilon$-matrices for all the cases discussed above). However, for a general two- or three-dimensional scattering problem, and inhomogeneities of irregular shapes, this procedure becomes cumbersome and the whole algorithmic simplicity of the MRTD method is lost. Based on the work in $[3,4]$, we propose the approximation of the $\varepsilon$-matrices by keeping only their diagonal elements. This greatly simplifies the MRTD formulation by decoupling the update equations. The quality of this approximation depends on the relative magnitude of the off-diagonal elements. In reference to Figs. 2 and 3, we can make the following observations: (a) the off-diagonal terms are larger when the contrast between the two media is greater; (b) the largest off-diagonal term is minimal when the interface is placed exactly half-way between two grid points. Also, the approximation made by truncating the off-diagonal elements is better as the size of the matrix is smaller. This is another reason for choosing scaling/wavelet functions with minimal support, like the CDF $(2,2)$ family (as opposed to higher-order CDF or Battle-Lemarie wavelet families). The resulting MRTD update scheme involves the equations in (7), where $\varepsilon$ is replaced by the diagonal elements of the $\varepsilon$-matrices (computed according to (10)). Such a scheme resembles the classic FDTD algorithm, where the material properties are sampled pointwise at the current grid point. This approach can be justified 
by the fact that, in the limit of very small discretization steps, the scaling functions behave like delta impulses.

In order to quantify the errors made by the approximation described above, we studied scattering by canonical dielectric targets, with different permittivity contrasts with respect to the background medium. The reference solution was obtained by the FDTD algorithm with a sampling rate of at least 12 points-per-wavelength at the smallest wavelength present in the computational domain. The CDF (2,2)-MRTD solution studied involved a scaling function expansion only, and a discretization rate half of that used by the FDTD algorithm (based on the dispersion curves in [5]). We measured the relative error of the far-zone frequency response magnitude, over a wide band of frequencies. Our results show that, up to a contrast of 4:1 in permittivity, the errors made in the CDF $(2,2)$-MRTD implementation are typically under $3 \%$, and even when the contrast was increased up to $12: 1$, the errors did not exceed $7 \%$ (the results are generally very good as a function of frequency, with most of the discrepancy seen in the frequency-localized nulls of the scattered spectrum).

The errors introduced by a diagonal approximation to the permittivity matrix do not increase simply with increasing dielectric contrast. In particular, as the dielectric contrast increases the scaling-function support decreases (in order to maintain the same sampling rate per wavelength inside the dielectric). As indicated above, the accuracy of the diagonal approximation improves with decreasing scaling-function support (since the scaling function better represents delta-function-like sampling). Therefore, the expected increased error due to increased dielectric contrast is mitigated by the reduced size of the scaling functions. We investigate these issues further in Sec. VI, where we present some numerical results.

\section{Absorbing boundary conditions}

The absorbing boundary conditions (ABC) for the MRTD algorithm can be implemented as a perfectly matched layer (PML) [6]. Since the PML consists of several successive layers with different material properties, a rigorous implementation within the MRTD algorithm would 
involve large $\varepsilon$ - and $\sigma$-matrices, reflecting the mutual coupling between all the scaling/wavelet functions corresponding to those layers. However, the implementation can be simplified using the approximations discussed in the previous section, that is, neglecting the coupling between update equations corresponding to adjacent layers. Our tests show no significant difference in the reflection coefficient between the rigorous and approximate implementations of the PML.

We also need to address the outer boundary of the computational grid, which is traditionally terminated by a perfect electric (or magnetic) conductor (PEC or PMC). Since updating the fields at points close to the boundary requires some field coefficients outside the domain, we can use image theory in order to obtain these coefficients. However, image theory becomes extremely complicated in the presence of a layered medium next to the boundary, and therefore its rigorous application is not practical. Instead, we notice that, if the PML is thick enough (compared to the stencil size), the magnitude of the field coefficients close to the boundary is very small and therefore errors made in computing these terms do not have a significant impact on the reflection coefficient. Our tests on a PML of just eight layers, in the context of a CDF (2,2)-MRTD two-dimensional implementation, show reflection coefficients no more than $-70 \mathrm{~dB}$, when the field coefficients outside the computational domain were simply set to zero.

\section{Numerical Results}

In this section we present the results of two-dimensional simulations obtained with the CDF (2,2)-MRTD scheme on scattering by dielectric targets and compare them with the FDTD solutions. The configuration is described in Fig. 4, and consists of two dielectric rectangular cylinders placed in free-space at a relatively large distance with respect to each other. For the MRTD implementation we use wavelet functions only in the shaded areas around the targets. This kind of configuration, in which targets are placed at relatively large distances from one

another in a homogeneous medium, is particularly suitable for the MRTD algorithm, because the coverage with high-resolution basis functions is modest compared to the entire computational 
domain. Therefore, significant savings in terms of computer memory can be obtained as compared to the Yee algorithm using a uniform mesh.

The excitation consists of a pulsed plane wave, with the incident waveform given by the $4^{\text {th }}$ order Rayleigh pulse, centered at $3 \mathrm{GHz}$ (Fig. 5). We consider TE (horizontal) polarization. The incidence angle is $45^{\circ}$ and the observation is made in the backscatter direction, in the far zone. We consider three permittivities for the targets: $\varepsilon_{r}=2,4,8$. For FDTD, we use a discretization rate of $40 \sqrt{\varepsilon_{r}}$ samples per central wavelength $\left(\lambda_{\mathrm{c}}\right)$ in air and a Courant number of 0.6. For the CDF-MRTD, we use a grid with $10 \sqrt{\varepsilon_{r}}$ samples per central wavelength in air. The Courant number is 0.3 , therefore the time step is twice as large as for FDTD. The wavelets cover about $12 \%$ of the MRTD computational domain, so the total number of scaling and wavelet coefficients is about 1.36 times the total number of MRTD cells. This means that we expect MRTD to utilize about 12 times less memory than FDTD, and to run about 6 times faster. However, our numerical experiments show that the increase in computational speed is more significant (typically, about 11 times). We attribute this to the fact that the MRTD update equations are more efficiently processed on the particular type of computer (Pentium III) that we used in our simulations. The MRTD dielectric matrices are approximated as diagonal, as discussed in Sec. VB.

The resulting waveforms, plotted in Figs. 6-8 show very good agreement between the two methods, even for permittivity contrasts as high as 8:1. This validates our approximations described in Sec. VB, and also illustrates the clear advantage of the method versus the traditional FDTD algorithm in terms of computational resources, for this kind of application.

\section{Conclusions}

In this paper we compared the MRTD algorithms based on certain families of compactly supported wavelets and applied them to the analysis of electromagnetic scattering problems. The low-order orthogonal Daubechies and biorthogonal CDF wavelet systems offer good 
computational efficiency, especially because of their low stencil size and the high Courant stability limit (the latter enabling the choice of a large time step). We also formulated an algorithm for the calculation of the MRTD coefficients, which avoids the numerical integration utilized by previous authors. We concluded that the schemes based on Daubechies- $(M+2)$ and $\operatorname{CDF}(2, M)$ basis functions have very similar performances, although the formulations are not identical. The discussion on applying the CDF $(2, M)$-MRTD to a scattering analysis included the implementation of the plane-wave incident field, material inhomogeneities and the absorbing boundary conditions, as well as numerical examples. We demonstrated that the approximation made in the treatment of dielectric boundaries keeps the implementation simple and efficient and, at the same time, yields accurate results. The computational savings of the CDF (2,2)-MRTD scheme versus the traditional FDTD method were clearly demonstrated in a numerical example. In future work, we will extend these MRTD schemes to three-dimensional problems.

\section{References}

[1] M. Krumpholz, L. Katehi, "MRTD: New time-domain schemes based on multiresolution analysis," IEEE Trans. on Microwave Theory and Techniques, vol. MTT-44, pp. 555-571, April 1996.

[2] T. Dogaru and L. Carin, "Application of multiresolution time-domain schemes to twodimensional electromagnetic scattering problems," submitted to IEEE Trans. Antennas and Propagation.

[3] Y.W. Cheong, Y.M. Lee, K.H. Ra, J.G. Kang, and C.C. Shin, "Wavelet-Galerkin scheme of time-dependent inhomogeneous electromagnetic problems," IEEE Microwave Guided Wave Letts., vol. 9, pp. 297-299, Aug. 1999.

[4] M. Fujii and W.J.R. Hoefer, "Dispersion of time domain wavelet Galerkin method based on Daubechies' compactly supported scaling functions with three and four vanishing moments," IEEE Microwave Guided Wave Letts., vol. 10, pp. 125-127, April 2000.

[5] T. Dogaru and L. Carin, "Multiresolution time-domain analysis using CDF biorthogonal wavelets," to appear in IEEE Trans. Microwave Theory and Techniques, vol. 49, May 2001.

[6] A. Taflove, Computational Electrodynamics: The Finite-Difference Time-Domain Method, 
Artech, Norwood, MA, 1995.

[7] G. Beylkin, "On the representation of operators in bases of compactly supported wavelets", SIAM J. Numer. Analysis, vol. 6, pp. 1716-1740, Dec. 1992.

[8] I. Daubechies, “Ten lectures on wavelets,” SIAM Rev., Philadelphia, PA, 1992 


\begin{tabular}{|c|c|c|c|c|c|c|c|}
\hline$i$ & -3 & -2 & -1 & 0 & 1 & 2 & 3 \\
\hline$\alpha$ & -0.1250000 & 0 & 1.1250000 & 2.0000000 & 1.1250000 & 0 & -0.1250000 \\
\hline$\beta$ & 0.1250000 & 0 & -1.1250000 & 2.0000000 & -1.1250000 & 0 & 0.1250000 \\
\hline$\gamma$ & -0.4665064 & 0 & 0.9665064 & 0 & -0.5334936 & 0 & 0.0334936 \\
\hline$\delta$ & 0.0334936 & 0 & -0.5334936 & 0 & 0.9665064 & 0 & -0.4665064 \\
\hline
\end{tabular}

(a)

\begin{tabular}{|c|c|c|c|c|c|c|}
\hline$i$ & -2 & -1 & 0 & 1 & 2 & 3 \\
\hline$r$ & -0.0833333 & 0.6666667 & 0 & -0.6666667 & 0.0833333 & 0 \\
\hline$a$ & -0.0104167 & 0.0937500 & -1.2291667 & 1.2291667 & -0.0937500 & 0.0104167 \\
\hline$b$ & 0.0104167 & -0.0937500 & 1.437500 & -1.437500 & 0.0937500 & -0.0104167 \\
\hline$c$ & -0.0388755 & 0.0805422 & -0.0055823 & -0.0777511 & 0.0444578 & -0.0027911 \\
\hline$d$ & 0.0027911 & -0.0444578 & 0.0777511 & 0.0055823 & -0.0805422 & 0.0388755 \\
\hline
\end{tabular}

(b)

\begin{tabular}{|c|c|c|c|c|c|c|c|c|c|}
\hline$i$ & -4 & -3 & -2 & -1 & 0 & 1 & 2 & 3 & -4 \\
\hline$\alpha$ & 0 & -0.1250 & 0 & 1.1250 & 2.0000 & 1.1250 & 0 & -0.1250 & 0 \\
\hline$\beta$ & 0 & 0.1250 & 0 & -1.1250 & 2.0000 & -1.1250 & 0 & 0.1250 & 0 \\
\hline$\gamma$ & 0.0625 & 0 & -1.0000 & 0 & 1.8750 & 0 & -1.0000 & 0 & 0.0625 \\
\hline$\delta$ & 0 & 0 & -0.2500 & 0 & 0.5000 & 0 & -0.2500 & 0 & 0 \\
\hline
\end{tabular}

(c) 


\begin{tabular}{|c|c|c|c|c|c|c|c|}
\hline$i$ & -3 & -2 & -1 & 0 & 1 & 2 & 3 \\
\hline$r$ & 0 & -0.0833333 & 0.6666667 & 0 & -0.6666667 & 0.0833333 & 0 \\
\hline$a$ & 0 & -0.0104167 & 0.0937500 & -1.2291667 & 1.2291667 & -0.0937500 & 0.0104167 \\
\hline$b$ & 0 & 0.0104167 & -0.0937500 & 1.437500 & -1.437500 & 0.0937500 & -0.0104167 \\
\hline$c$ & 0.0052083 & -0.0833333 & 0.1510417 & 0 & -0.1510417 & 0.0833333 & -0.0052083 \\
\hline$d$ & 0 & -0.0208333 & 0.0416667 & 0 & -0.0416667 & 0.0208333 & 0 \\
\hline
\end{tabular}

(d)

Table I. (a) Correlation coefficients for the Daubechies-4 wavelet family; (b) MRTD coefficients for the Daubechies-4 scheme; (c) Correlation coefficients for the CDF (2,2) wavelet family; (d) MRTD coefficients for the CDF $(2,2)$ scheme.

\begin{tabular}{|c|c|c|c|c|c|c|}
\hline & Daub-4 & Daub-6 & Daub-8 & CDF (2,2) & CDF (2,4) & CDF (2,6) \\
\hline$n_{a}$ & 3 & 5 & 7 & 3 & 5 & 7 \\
\hline$n_{b}$ & 3 & 5 & 7 & 3 & 5 & 7 \\
\hline$n_{c}$ & 3 & 5 & 7 & 3 & 6 & 9 \\
\hline$n_{d}$ & 3 & 5 & 7 & 2 & 3 & 4 \\
\hline
\end{tabular}

Table II. Stencil sizes for Daubechies- $(M+2)$ and CDF $(2, M)$ MRTD schemes. 


\section{Figure Captions}

Figure 1. Phase error (in degrees per wavelength) vs. discretization rate for MRTD schemes using expansion of the fields in terms of scaling and one level wavelet functions. For all schemes, the Courant number is $95 \%$ of the stability limit. One-dimensional propagation.

Figure 2. Support of the CDF $(2,2)$ scaling and dual scaling basis functions involved in coupling through a permittivity discontinuity. The discontinuity is placed at a grid point. Note that the supports of the scaling/dual scaling functions are drawn schematically - they do not represent the graphs of the actual basis functions.

Figure 3. Support of the CDF $(2,2)$ scaling and dual scaling basis functions involved in coupling through a permittivity discontinuity. The discontinuity is placed in between grid points. Note that the supports of the scaling/dual scaling functions are drawn schematically - they do not represent the graphs of the actual basis functions.

Figure 4. Computational domain for the numerical example in Section VI. It consists of two rectangular dielectric cylinders, placed in free-space. The central wavelength is $\lambda_{c}=10 \mathrm{~cm}$.

Figure 5. The incident pulse (Rayleigh, $4^{\text {th }}$ order) in (a) time and (b) frequency domain. The central frequency is $3 \mathrm{GHz}$.

Figure 6. Time-domain scattered field for the configuration in Fig. 4 and $\varepsilon_{r}=2$. The two curves are almost identical.

Figure 7. Time-domain scattered field for the configuration in Fig. 4 and $\varepsilon_{r}=4$. The two curves are almost identical.

Figure 8. Time-domain scattered field for the configuration in Fig. 4 and $\varepsilon_{r}=8$. The two curves are almost identical. 


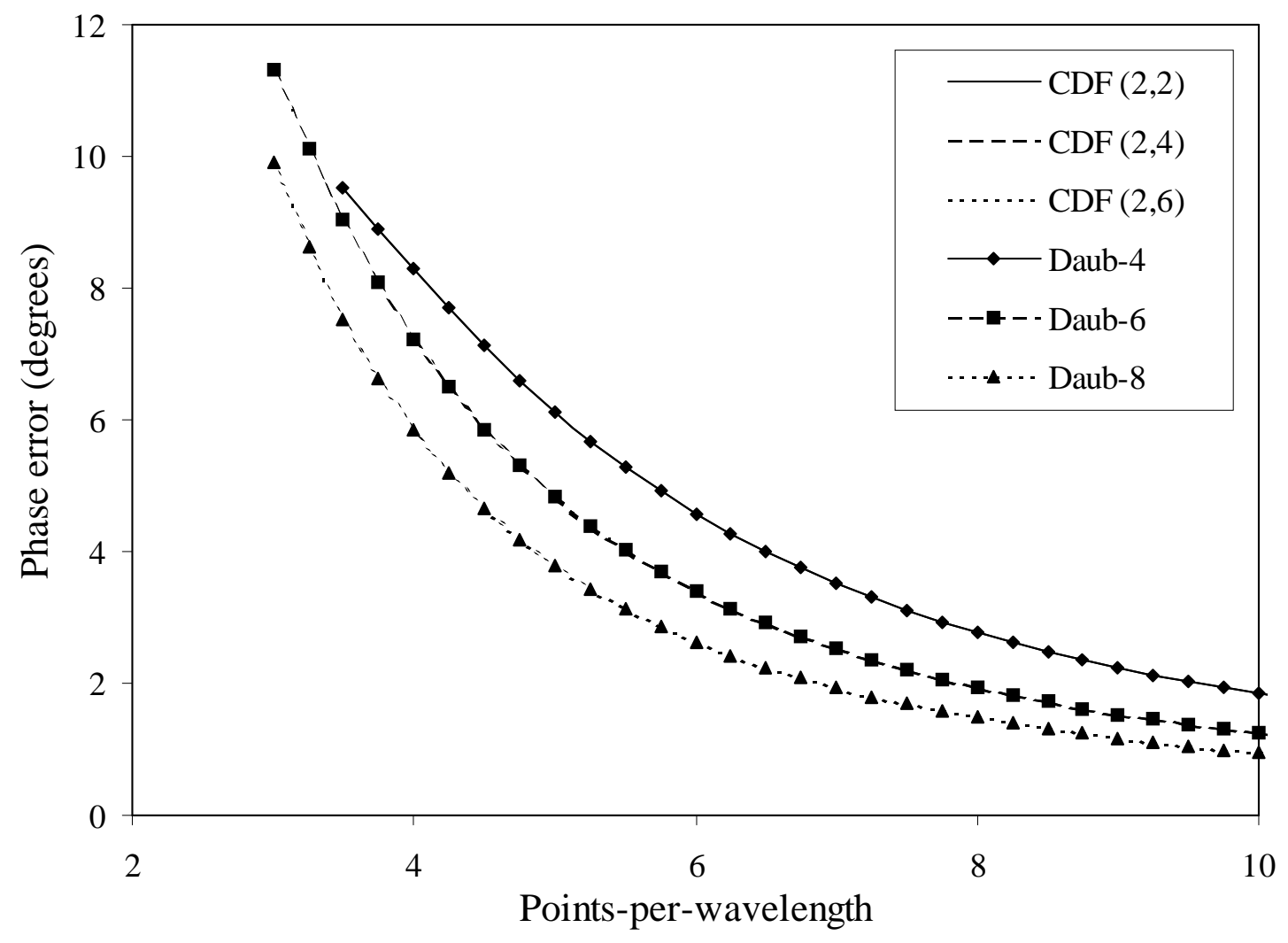

Figure 1 


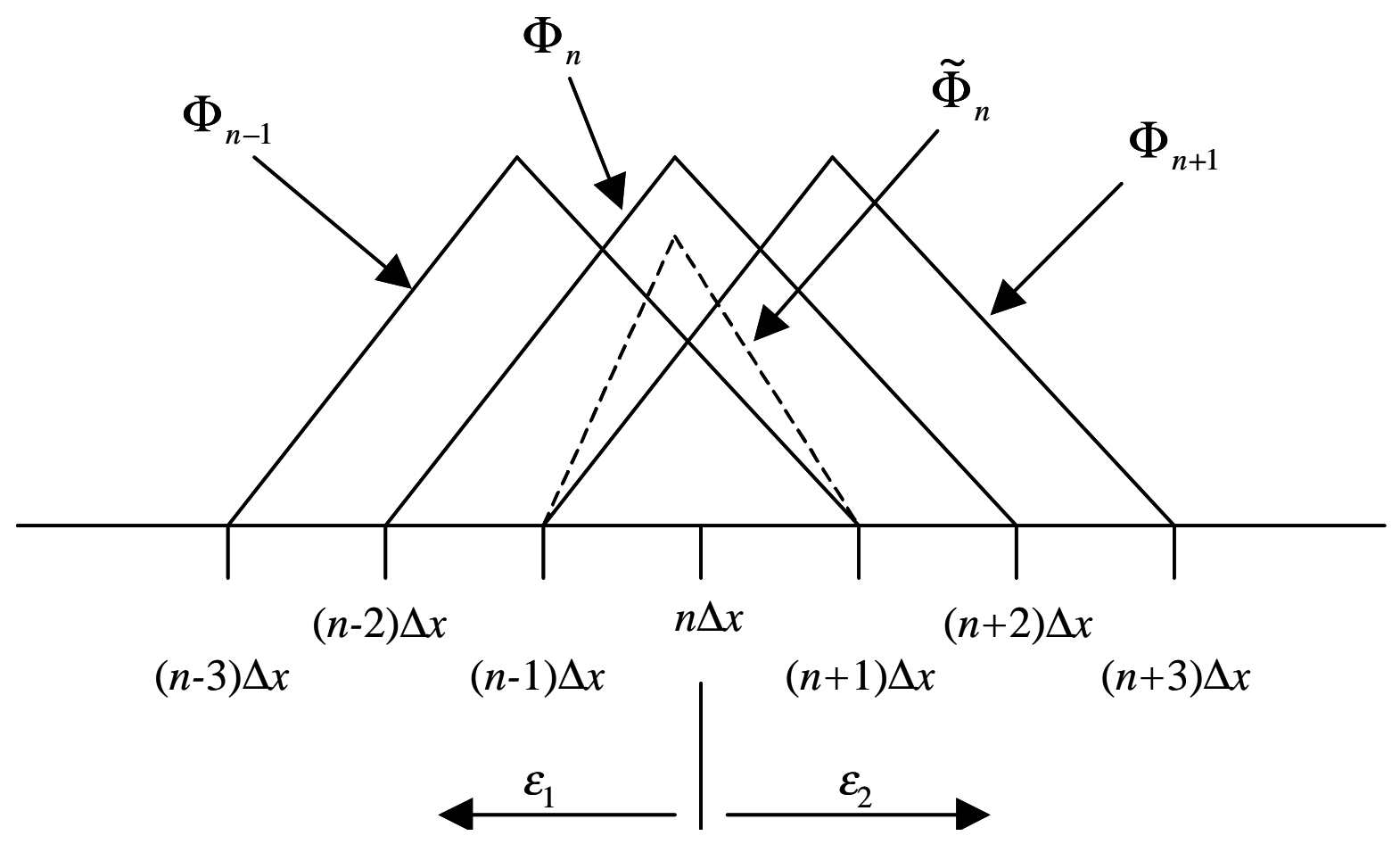

Figure 2

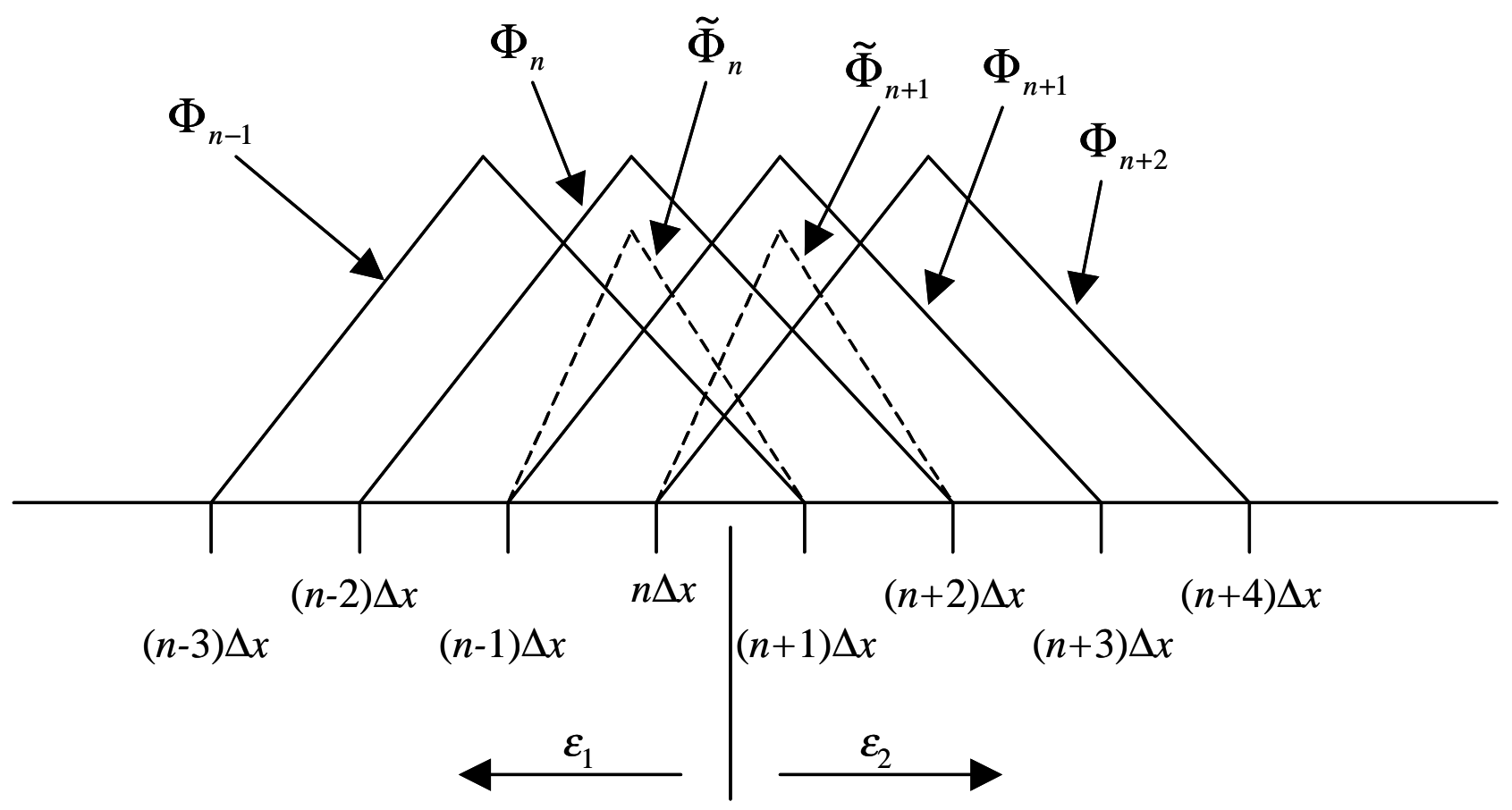

Figure 3 


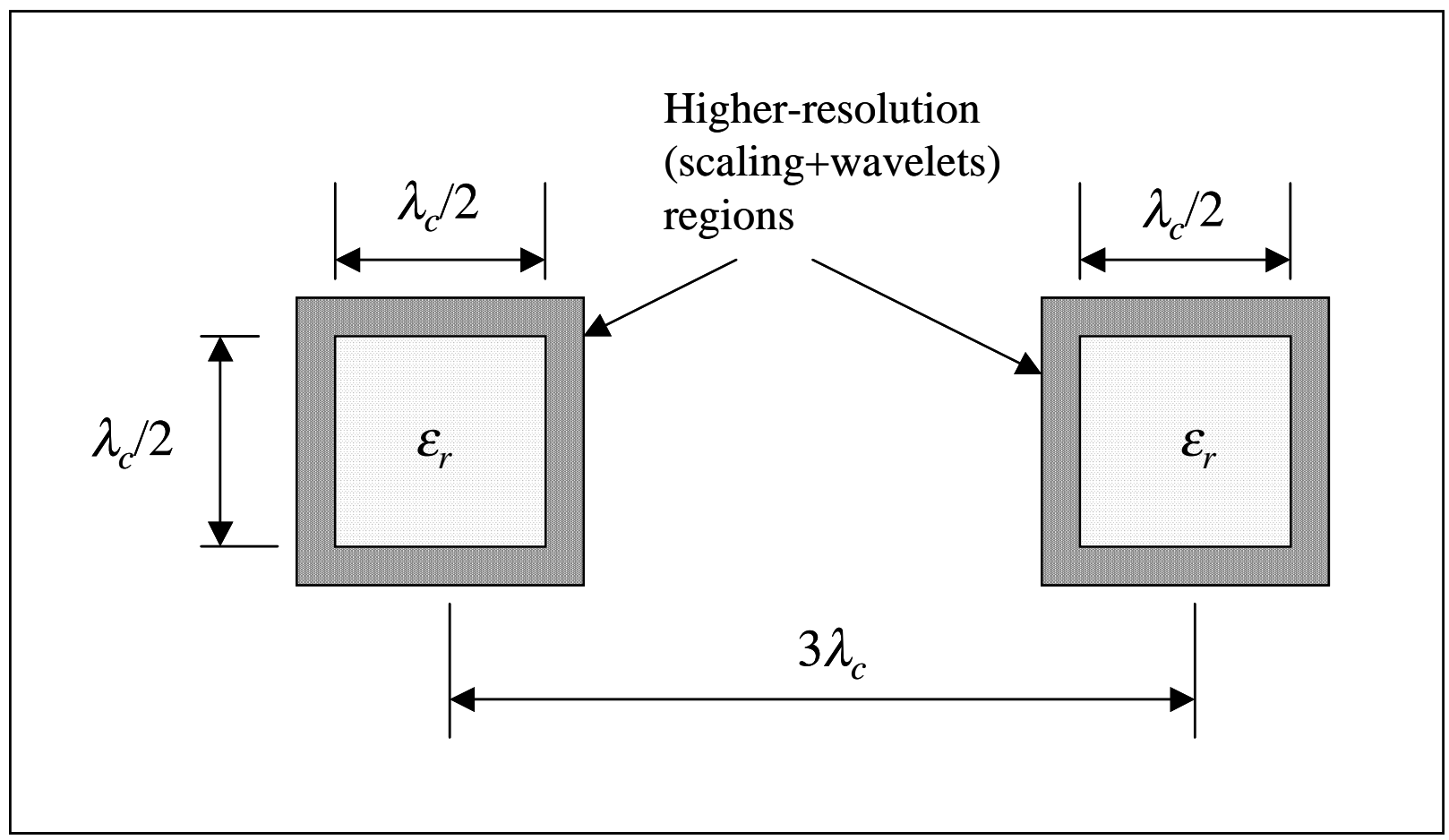

Figure 4

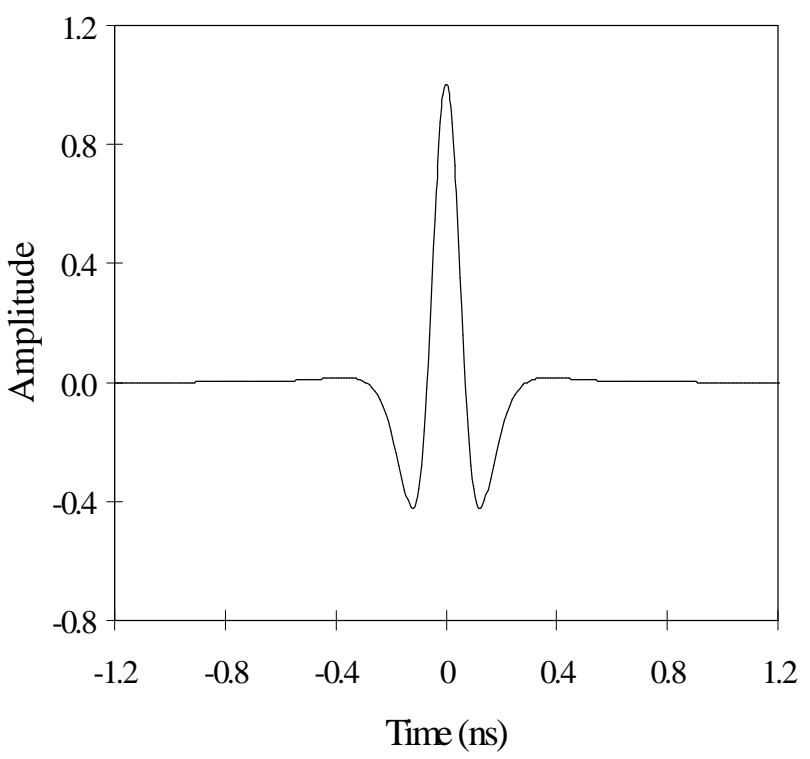

(a)

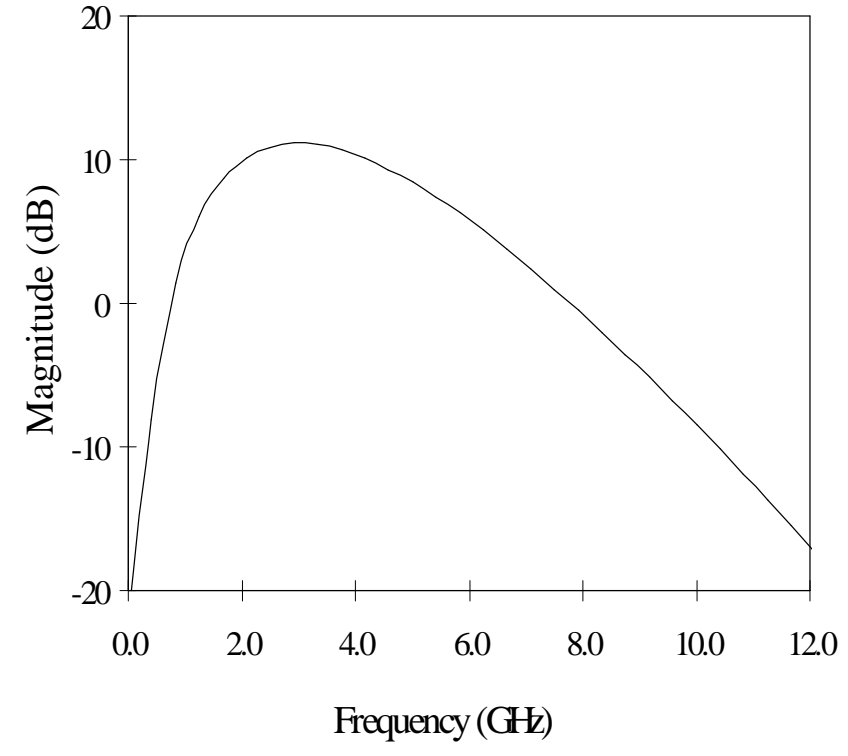

(b)
Figure 5 


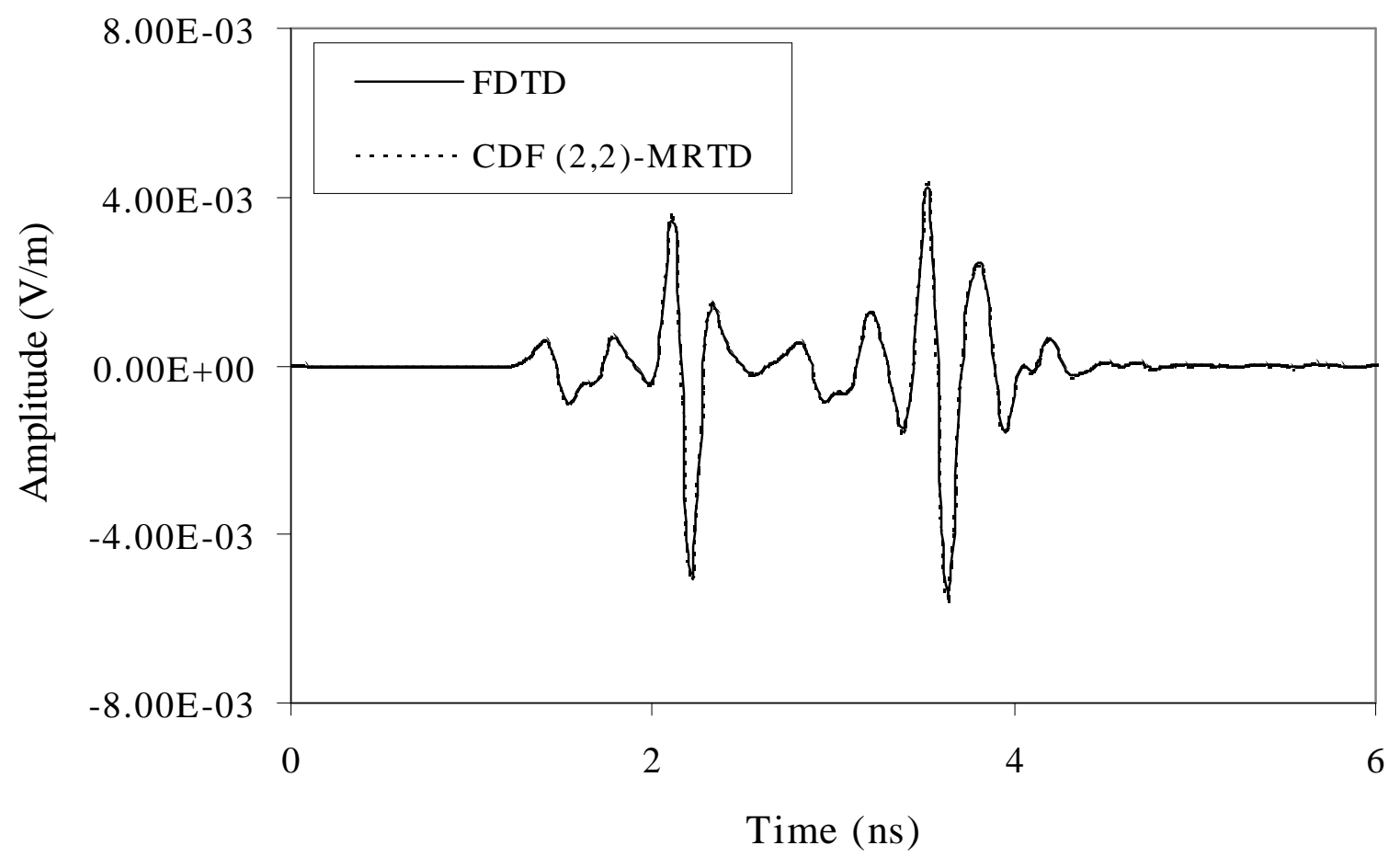

Figure 6

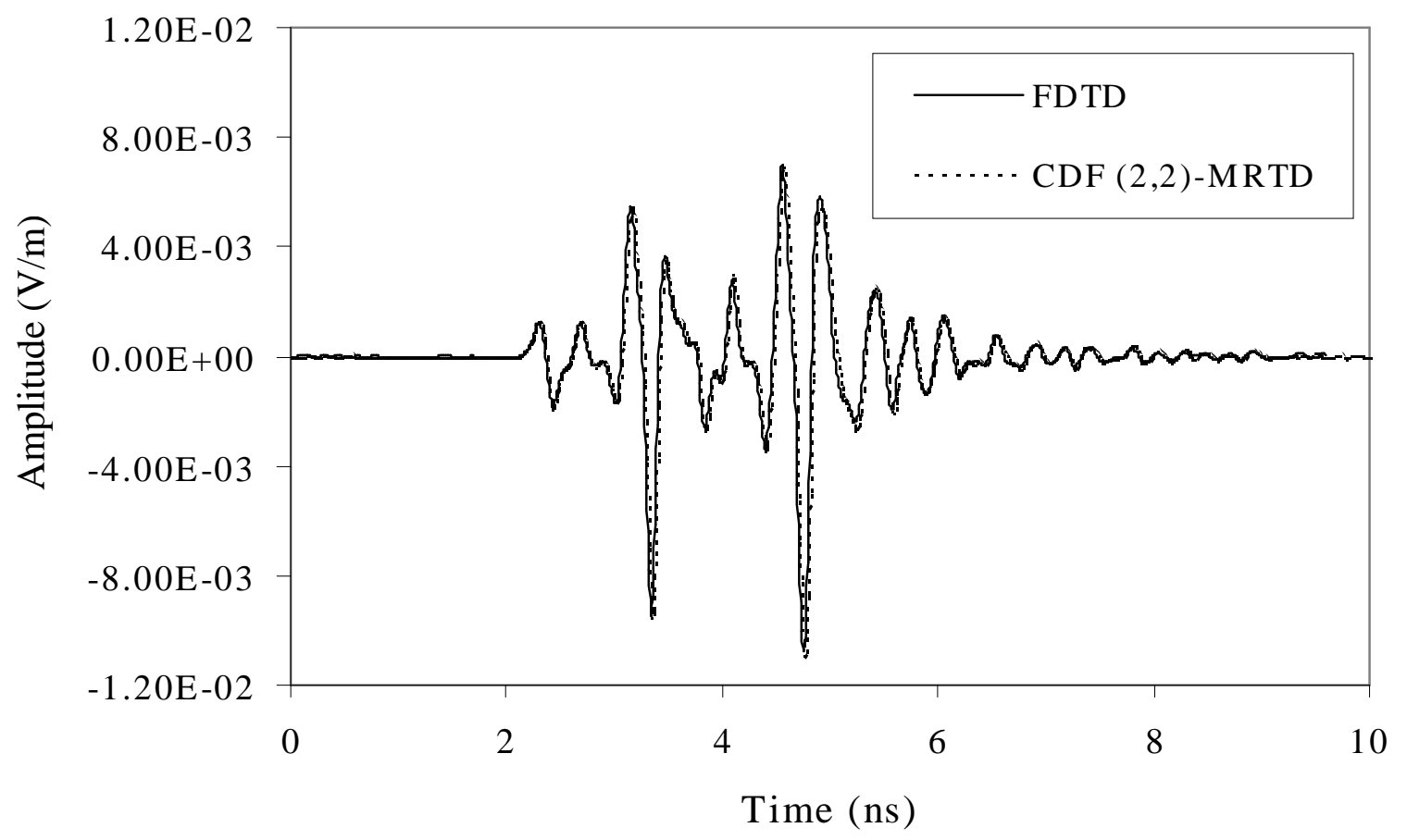

Figure 7 


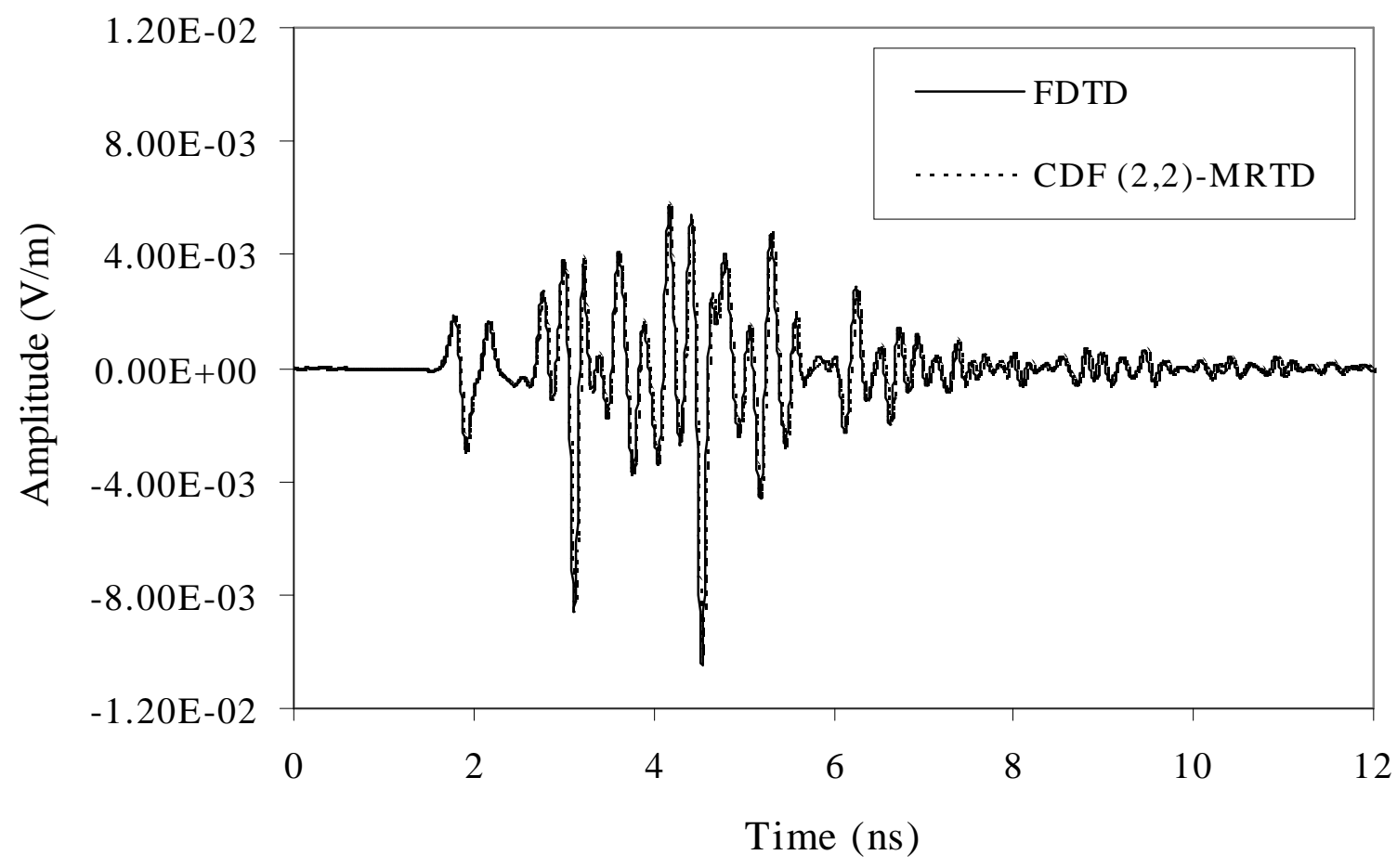

Figure 8 